\title{
Comparison of Platelet Indices with Platelet Count to Predict Bleeding Tendency in Seropositive Dengue Cases
}

\author{
Authors \\ Sudhakar Ramamoorthy ${ }^{1}$, Poongodi Rajagopal ${ }^{2}$, Prasanna Rajguru ${ }^{3}$ \\ ${ }^{1,2}$ Assistant Professor, Department of Pathology, Velammal Medical College Hospital and Research \\ Institute, Madurai \\ ${ }^{3}$ Undergraduate, Velammal Medical College Hospital and Research Institute, Madurai \\ Corresponding Author
}

Dr Sudhakar Ramamoorthy

Assistant Professor, Department of Pathology, Velammal Medical College Hospital and Research Institute, Madurai

Email: dr.sudhakar2k3@gmail.com

\begin{abstract}
Objective: To compare platelet indices with platelet count in predicting bleeding tendencies of dengue positive cases.

Materials and Methods: The study was carried out in a tertiary care hospital. 50 seropositive dengue patients were enrolled in the study. Clinical presentations and bleeding signs were monitored. Platelet count, mean platelet volume, platelet distribution width and plateletcrit were measured on the day of bleeding and on the day patient recovered from bleeding manifestations.

Results: In the present study, the chief presenting complaint was fever (92\%) which is followed by vomiting, abdominal pain and bleeding. Malena was identified as the common bleeding sign seen in $48 \%$ of the patients. Study group showed a marked decrease in plateletcrit value during bleeding $0.07 \pm 0.05 \%$ when compared to the platelecrit values during recovery $0.13 \pm 0.04 \%$ and was statistically significant similar to platelet count. However, the significance was much higher for plateletcrit $(p=0.0001)$ compared to platelet count $(p=0.001)$. Mean platelet volume and platelet distribution width showed no significant difference between bleeding and recovery periods.

Conclusion: The present study showed a significant decrease in plateletcrit and platelet count when the patient developed bleeding manifestations and progressively increases during the period of recovery. The rise in value during recovery is prominent for plateletcrit when compared to platelet count. Of all the platelet indices, plateletcrit can be used as an adjunct to platelet count to predict bleeding and recovery.

Keywords: Dengue, plateletcrit, mean platelet volume, platelet distribution width, platelet count, bleeding.
\end{abstract}

\section{Introduction}

Dengue is a major public health problem and a leading cause of hospitalization among children. The disease is prevalent in India for last five decades with hyper endemicity for all its four serotypes. Dengue has a wide clinical spectrum ranging from asymptomatic phase to dengue fever, dengue hemorrhagic fever and dengue shock syndrome. Dengue fever usually involves older children and adults resulting in a biphasic fever, 
headache, vomiting, myalgia and bone pain. Dengue hemorrhagic fever is more common in children less than 15 years of age, in endemic regions and in repeated dengue infections. In addition, the patient presents with one or more of the hemorrhagic manifestations that include petechiae, purpura (venipuncture sites), ecchymosis, epistaxis, gum bleeding, haematemesis, melena or a positive tourniquet test. Besides, the patient present with warning signs like abdominal pain, continuous vomiting, lethargy, irritability and oliguria which precede shock. Dengue shock syndrome include the features of dengue hemorrhagic fever along with organ failure due to plasma leakage into serous cavities. In dengue shock syndrome, patients present with shock in the form of weak pulse, cold clammy skin and narrowed pulse pressure $(<20 \mathrm{mmHg})$. Of all the clinical features, the disease severity and mortality depends upon bleeding manifestations and shock. The bleeding is caused by thrombocytopenia, vasculopathy and coagulopathy. ${ }^{1}$ According to the WHO guidelines 2011, thrombocytopenia is used as the main parameter to grade dengue hemorrhagic fever. The platelet count has also been used as a predictor of bleeding, a marker of severity and a criteria for patient recovery. ${ }^{2,3}$ Thus platelet count has become an essential tool in every phase of dengue infections. But there are certain limitations in using platelet count as an individual parameter. Hemostasis depends not only on platelet count but also on platelet activity. False low platelet count has also been noticed in routine practice due analytical errors of automated analysers when there are giant platelets and platelet aggregates. Such erroneous results may lead onto unnecessary transfusions. Irrespective of the limitations, attention was given only to the Platelet count which concealed the usefulness of Platelet indices like Plateletcrit (PCT), Mean Platelet Volume (MPV) and Platelet Distribution Width(PDW).

PCT is an indicator of circulating platelets in an unit volume of blood. MPV is a measure of platelet volume. PDW measures the variability in size of platelets. In dengue, thrombocytopenia occurs mainly due to immune mediated platelet destruction. ${ }^{4}$ To compensate these loss, bone marrow produces young large volume platelets which leads to high MPV. Platelets with high MPV and high PDW were found to be the markers of platelet activation thus enabling a high hemostatic property irrespective of the degree of thrombocytopenia. ${ }^{5}$ However PDW is more specific than MPV as a platelet activation marker since it will not be influenced by platelet swelling. ${ }^{6}$ It should be remembered that the main dreadful complication of dengue was not by the disease per se, but by excessive unnecessary transfusions that will result in congestive cardiac failure. To also avoid such circumstances, platelet count should be accompanied by some other parameters like mean platelet volume, platelet distribution width and plateletcrit.

Based on these concepts regarding platelet indices, our study was formulated to know whether platelet indices can be used as a better alternative or an adjunct to platelet count in evaluating bleeding tendencies and to assess recovery.

\section{Materials and Methods}

The study included 50 consecutive seropositive dengue cases admitted in a tertiary care hospital. Clinically suspected dengue cases were confirmed on serology by immunochromatographic method (SD Bioline Dengue Duo, Korea). The cases seropositive for NS1 or IgM or both were included in the study (Indicative of recent infection). All these cases were subjected to thorough clinical examination including bleeding manifestations.

A patient with any one or more of the following signs were considered to be having a bleeding tendency

\section{Petechiae}

2.Purpura (venipuncture sites)

3.Ecchymosis

4.Epistaxis

5.Gum bleeding

6.Haematemesis

7.Melena

8.Positive tourniquet test (The tourniquet test were performed by inflating a blood pressure cuff to a point midway between the systolic and diastolic 


\section{JMSCR Vol||05||Issue||12||Page 31638-31643||December}

pressures for five minutes. The test were considered positive if 10 or more petechiae per square inch are observed).

Platelet count and platelet indices (MPV,PCT and PDW) of these patients were noted from the data stored in our Beckman LH 750 hematology analyser computer system. All these four platelet parameters were measured at two periods of hospital stay namely, on the day patients develop bleeding signs and on the day when patient recovered from bleeding manifestations.

\section{Exclusion criteria}

1. Patients positive for only $\operatorname{IgG}$ serology were excluded from the study. Since IgG is a marker of past infection, this may not reflect the current status of the patient.

2. Dengue positive patients who received prophylactic blood transfusions before the emergence of bleeding manifestations were excluded from the study as it will intervene in platelet values.

\section{Results}

During the study period a total of 50 cases of seropositive dengue cases were analyzed. Out of 50 seropositive cases, 25 cases have associated bleeding signs and 25 cases did not have any associated bleeding sign. Of the 25 cases who had bleeding signs, 10 cases presented with bleeding sign on the day of admission and remaining 15 cases manifested bleeding during the hospital stay. The most frequent presenting complaint of the study population was fever, followed by vomiting, abdominal pain and bleeding (Figure 1). Of the different bleeding signs identified in dengue patients, the first two common were melena and petechial hemorrhages (Figure 2).

Table 1 : Comparison of platelet count and platelet indices in dengue patients

\begin{tabular}{|c|c|c|c|c|c|c|c|c|}
\hline \multirow[t]{2}{*}{ S.No } & \multicolumn{2}{|c|}{ Platelet count(cells/cumm) } & \multicolumn{2}{|c|}{ MPV (fl) } & \multicolumn{2}{|c|}{ PDW (fl) } & \multicolumn{2}{|c|}{ PCT $(\%)$} \\
\hline & Day of bleeding & $\begin{array}{c}\text { Day of } \\
\text { discharge }\end{array}$ & $\begin{array}{c}\text { Day of } \\
\text { bleeding }\end{array}$ & $\begin{array}{c}\text { Day of } \\
\text { discharge }\end{array}$ & Day of bleeding & $\begin{array}{c}\text { Day of } \\
\text { discharge }\end{array}$ & Day of bleeding & $\begin{array}{c}\text { Day of } \\
\text { discharge }\end{array}$ \\
\hline 1. & 12000 & 154000 & 8.5 & 10.4 & 18.2 & 16.8 & 0.09 & 0.11 \\
\hline 2. & 19000 & 85000 & 8.8 & 8.6 & 17.4 & 19.4 & 0.02 & 0.07 \\
\hline 3. & 16000 & 367000 & 7.3 & 6.8 & 17.4 & 17.7 & 0.01 & 0.25 \\
\hline 4. & 150000 & 184000 & 7.5 & 8.1 & 16.9 & 17.1 & 0.11 & 0.15 \\
\hline 5. & 31000 & 139000 & 9.3 & 10.7 & 16.6 & 18.5 & 0.03 & 0.15 \\
\hline 6. & 93000 & 163000 & 9.5 & 7.9 & 18.1 & 18.3 & 0.13 & 0.13 \\
\hline 7. & 11000 & 112000 & 9.3 & 8.6 & 17.6 & 18.1 & 0.01 & 0.1 \\
\hline 8. & 19000 & 143000 & 9.6 & 9.1 & 17.6 & 19.2 & 0.02 & 0.13 \\
\hline 9. & 20000 & 20000 & 9.1 & 8.1 & 16.4 & 18 & 0.02 & 0.16 \\
\hline 10. & 58000 & 30700 & 7 & 8.6 & 17.1 & 19.9 & 0.07 & 0.1 \\
\hline 11. & 258000 & 27600 & 7.3 & 6.8 & 16.6 & 16.3 & 0.19 & 0.19 \\
\hline 12. & 33000 & 58000 & 8.3 & 8.6 & 17.7 & 17.8 & 0.03 & 0.08 \\
\hline 13. & 30000 & 150000 & 8.5 & 6.1 & 16.5 & 16.9 & 0.03 & 0.11 \\
\hline 14. & 63000 & 120000 & 7.9 & 8.8 & 17 & 17.9 & 0.07 & 0.1 \\
\hline 15. & 30000 & 180000 & 9.1 & 8 & 17.5 & 16.2 & 0.03 & 0.14 \\
\hline 16. & 40000 & 110000 & 8.5 & 8.6 & 19.7 & 16.9 & 0.1 & 0.1 \\
\hline 17. & 25000 & 94000 & 9 & 8.6 & 16 & 17.7 & 0.03 & 0.1 \\
\hline 18. & 30000 & 150000 & 9.3 & 9.1 & 16.4 & 16.4 & 0.03 & 0.1 \\
\hline 19. & 47000 & 120000 & 13 & 8.8 & 19.5 & 17.7 & 0.06 & 0.1 \\
\hline 20. & 230000 & 210000 & 7.5 & 7.7 & 16.1 & 16 & 0.18 & 0.19 \\
\hline 21. & 34000 & 110000 & 8.6 & 8.9 & 17.1 & 17 & 0.03 & 0.1 \\
\hline 22. & 150000 & 130000 & 6.5 & 7.1 & 17.2 & 17.5 & 0.11 & 0.1 \\
\hline 23. & 10000 & 190000 & 7.6 & 7.5 & 18.2 & 16 & 0.11 & 0.17 \\
\hline 24. & 40000 & 140000 & 8 & 7.4 & 16.6 & 18.3 & 0.12 & 0.15 \\
\hline 25. & 20000 & 220000 & 8.8 & 7.3 & 17.4 & 16 & 0.02 & 0.11 \\
\hline
\end{tabular}

Platelet count and platelet indices of 25 patients with bleeding signs were analyzed separately. In these 25 cases, each platelet parameter on the day of bleeding were correlated with the same platelet parameter on the day of recovery. The mean value of platelet count on the day of bleeding was obviously low (58760 cells/cu.mm) in comparison to that measured on the day of recovery (136292 cells/cumm). Similarly the other three platelet parameters were compared during the day of 


\section{JMSCR Vol||05||Issue||12||Page 31638-31643||December}

bleeding and day of recovery (Table 1). Mean platelet volume and platelet distribution width showed no significant difference. Plateletcrit however, showed a markedly decreased value during bleeding phase and the values improved dramatically during recovery in 22 patients and unchanged in the remaining 3 patients. Wilcoxon signed rank test showed a statistically significant difference in platelet count $(\mathrm{p}=0.001)$ and plateletcrit $(\mathrm{p}=0.0001)$ (Table 2). ROC curve was plotted to know if there was any parameter which predict bleeding from the values recorded on the day of admission. However no value recorded on the day of admission was found to predict bleeding.

Table 2: Wilcoxon Signed Ranks test for comparison of platelet parameters during bleeding and recovery

\begin{tabular}{|l|c|c|c|c|}
\hline & $\begin{array}{c}\text { PC recovery - PC } \\
\text { bleeding }\end{array}$ & $\begin{array}{c}\text { MPV recovery - } \\
\text { MPV bleeding }\end{array}$ & $\begin{array}{c}\text { PDW recovery - } \\
\text { PDW bleeding }\end{array}$ & $\begin{array}{c}\text { PCT recovery - PCT } \\
\text { bleeding }\end{array}$ \\
\hline$Z$ & -3.429 & -1.037 & -1.015 & -4.106 \\
Asymp. Sig. (2-tailed) & .001 & .300 & .310 & .0001 \\
\hline
\end{tabular}

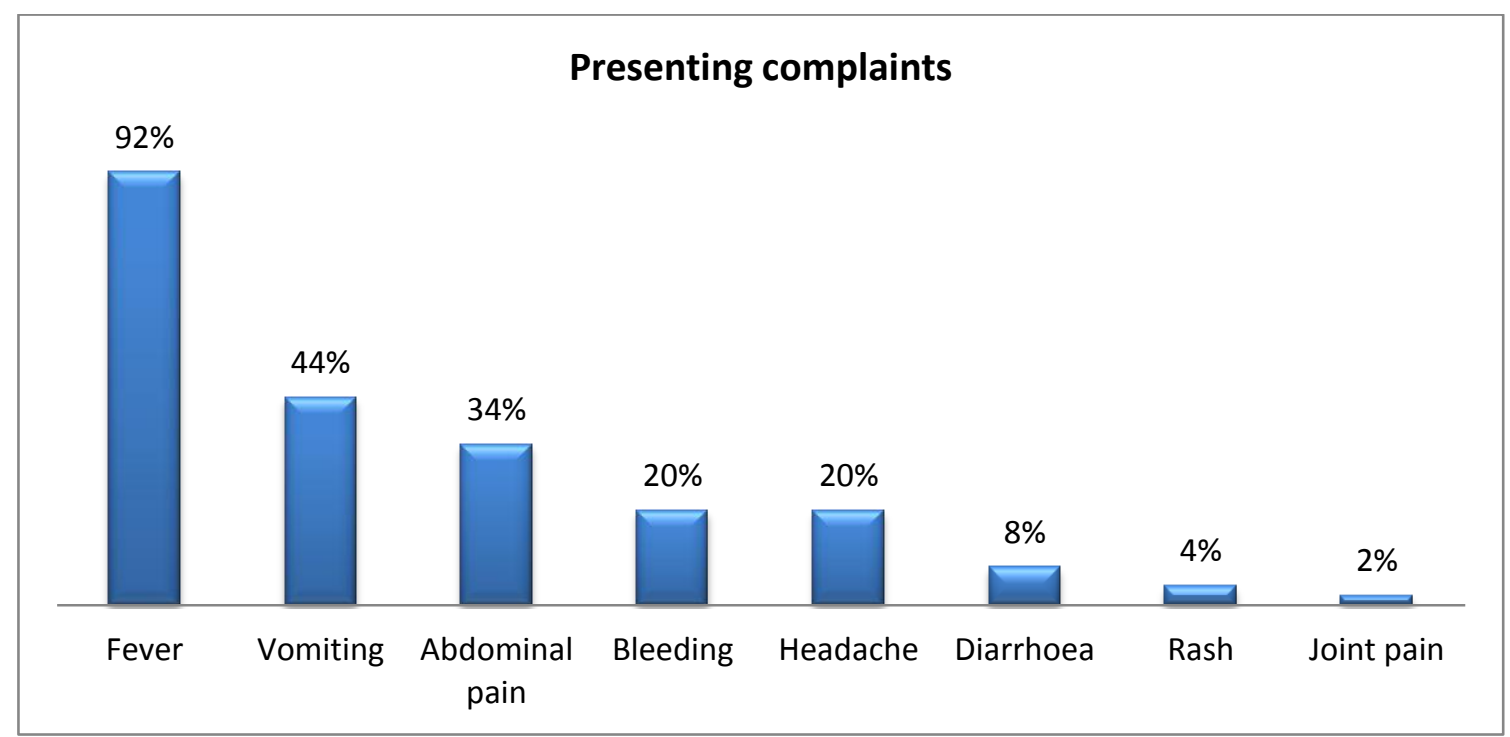

Figure 1: Frequency of presenting complaints in dengue cases

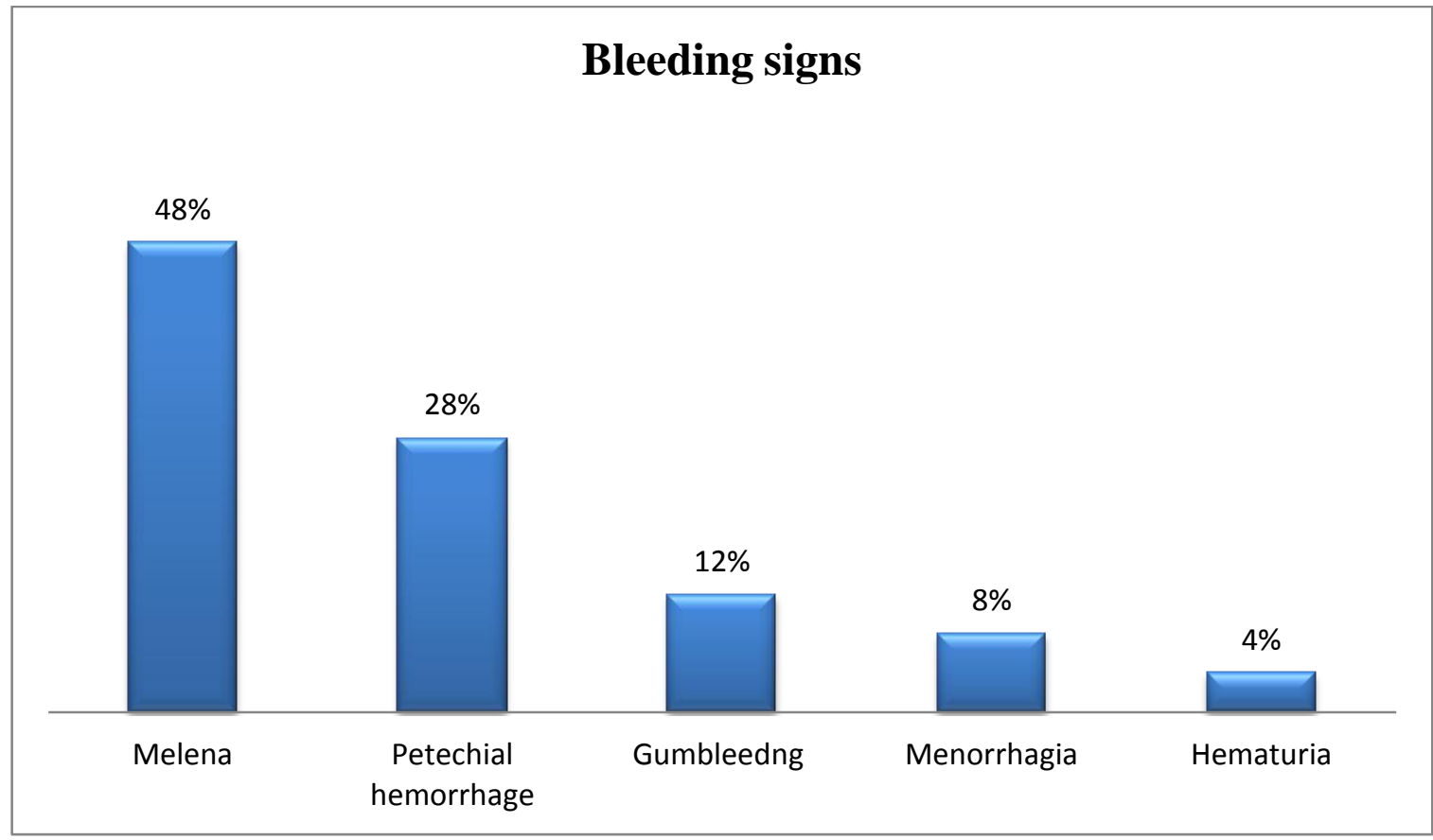

Figure 2 : Frequency of different bleeding signs in dengue patients. 


\section{Discussion}

Our present study showed that the bleeding manifestations in dengue is common in adults (62\%) compared to children (30\%). A comparable finding was noted in the study conducted by Joseph et al. The study explained the factor responsible for increased prevalence of dengue hemorrhagic fever in adults compared to children. Dengue infection in children were mostly primary, which are asymptomatic. Whereas in adults, the infections are secondary which are symptomatic and lead on to dengue fever and dengue hemorrhagic fever. ${ }^{7}$

In the present study, male predominance $(62 \%)$ was noted which is in comparable with the previous study conducted in six asian countries by anker et al. In addition, our study also showed that the bleeding tendencies are common in females (57\%) as compared to males. ${ }^{8}$

In our study we also found that fever $(92 \%)$ and vomiting $(44 \%)$ are the most common presenting illness. Among all bleeding manifestations, melena $(48 \%)$, petechial hemorrhages $(28 \%)$ are common . This finding might be partially comparable with a previous study, which stated that the main risk factor for mortality in dengue are hematemesis and melena. $^{9}$

Mean platelet count on day of bleeding in our study is 58760 cells/cumm of blood. This value is about half to one third of the value obtained on day of recovery. This signifies that thrombocytopenia has a direct correlation with bleeding tendency as expected. Platelet count significantly decreased in bleeding phase and improved during recovery which correlate with earlier studies. ${ }^{2}$ Thus platelet count can be used as a marker to predict bleeding and hence grade the severity of the disease. However a combination of markers is always efficient in predicting bleeding when compared with platelet count alone.

In addition to the platelet count, plateletcrit also showed a significant difference (0.0001). Plateletcrit were low on the day of bleeding (mean:0.07) than values on the day of recovery (Mean:0.13). In two of the patients where platelet count decreased during clinical recovery, plateletcrit improves thus correlating with the clinical findings. This implies that the factors that affect platelet count (Platelet aggregates and giant platelets) may not influence plateletcrit thus it reflects the invivo status of platelets precisely than the platelet count. Plateletcrit has already gained importance as a screening tool to diagnose platelet quantitative disorders in addition to platelet count. ${ }^{10}$ Similarly plateletcrit might be used along with platelet count to predict bleeding and grade severity in dengue cases.

No significant difference was noted in MPV and PDW values. Both MPV and PDW related with the platelet size, MPV measures size of the platelets and PDW measures variability in size of the platelets. A study by chuansumrit et al proposed that, thrombocytopenia in dengue are caused by immune mediated platelet destruction. ${ }^{4}$ This will result in large size platelet production by bonemarrow which increase MPV and PDW compared to control samples. However our study did not show any significant difference irrespective of thrombocytopenia. However a study by Sansanee et al, showed that dengue virus suppress bone marrow rather than peripheral destruction which is in contrast to the study by chuansumrit et al. If dengue virus suppress bone marrow as proposed by sansanee et al, there wont be any variation in platelet size. ${ }^{11}$ This will result in unchanged MPV and PDW values during bleeding as well as in recovery phases irrespective of thrombocytopenia which was identified in the present study.

To conclude, the present study suggests the incorporation of plateletcrit along with platelet count to predict bleeding and recovery in dengue patients. However, studies on a larger scale is required to develop cut-off levels of plateletcrit to predict bleeding tendencies in dengue patients precisely.

\section{Acknowledgements}

We would like to express our gratitude to Dr.Rizwan who performed the statistical analysis for the study 


\section{References}

1. Srichaikul T, Nimmannitya S. Haematology in dengue and dengue haemorrhagic fever. Baillieres Best Pract Res Clin Haematol. 2000; 13(2): 261-76.

2. Jayashree K, Manasa G C, Pallavi P, Manjunath G V. Evaluation of Platelets as Predictive Parameters in Dengue Fever. Indian J Hematol Blood Transfus.2011; 27(3):127-130.

3. Pongpan S, Wisitwong A, Tawichasri C, Patumanond J, Namwongprom S. Development of dengue infection severity score. ISRN Pediatr. 2013;2013:845876.

4. Chuansumrit A, Tangnararatchakit K. Pathophysiology and management of dengue hemorrhagic fever. Transfus Altern Transfus Med.2006;8(Suppl):3-11. doi: 10.1111/j.1778-428X.2006.00025.x

5. Vagdatli, Gounari, Lazaridou, Katsibourlia, Tsikopoulou, Labrianou. Platelet distribution width: a simple, practical and specific marker of activation of coagulation. Hippokratia. 2010;14(1):28- 32.

6. Dadu et al. Evaluation of the immature platelet fraction as an indicator of platelet recovery in dengue patients. Int $\mathrm{J}$ Lab Hematol. 2014 Oct;36(5):499-504.doi: 10.1111/ijlh.12177.

7. Joseph R. Egger, Paul G. Coleman.Age and Clinical Dengue Illness Emerg Infect Dis. 2007; 13(6): 924-927.

8. Anker $\mathrm{M}$ and Arima Y. Male-female differences in the number of reported incident dengue fever cases in six Asian countries. Western Pacific Surveillance and Response Journal. 2011;2(2):17-23.

9. Navarrete et al. Clinical profile of dengue hemorrhagic fever cases in Mexico. Salud Publica Mex. 2005;47(3):193-200.

10. Vani chandrashekar. Plateletcrit as a Screening Tool for Detection of Platelet
Quantitative Disorders. Journal of hematology.2013;2(1):22-26.

11. Sansanee et al. Infection of bone marrow cells by dengue virus in vivo. Exp Hematol. 2012; 40(3): 250-259. 